\title{
Metodología para la implementación del método adaptativo de Monte Carlo en la evaluación de la incertidumbre de la medición, utilizando el cálculo simbólico Maple. Aplicación a un experimento sencillo
}

\section{Gustavo Delgado ${ }^{1^{*}}$}

1. Universidad Nacional Autónoma de Nicaragua, León (UNAN-LEÓN). Facultad de Ciencias y Tecnología, Departamento de Química, Laboratorio de Análisis de Trazas de Metales Pesados (LATMP), Edificio de Ciencias Básicas, León, Nicaragua. Tel: (505) 2311 5013, ext: 1132. Fax: (505) 23114012.

\section{RESUMEN}

El objetivo del presente estudio es aplicar una metodología rigurosa para estimar la incertidumbre de las mediciones utilizando el método adaptativo de la simulación de Monte Carlo (MCM). Se tomó como ejemplo la estimación de la incertidumbre en la medición del área de un triángulo. El valor del área $(y)$ y su incertidumbre asociada $(u)$ se calcularon en base a un algoritmo que se programó en lenguaje Maple 12, generando un total de 10000 valores del mensurando. Para calcular el intervalo de confianza (o intervalo de cobertura), se exportaron estos valores a la hoja de cálculo MS Excel, se obtuvieron los porcentajes acumulados de la función de distribución de probabilidad (CPDF) y se evaluaron los valores extremos del intervalo de cobertura $\left(y_{\text {inf }}, y_{\text {sup }}\right)$ a una probabilidad del $95 \%$. A partir de los resultados se trazó el histograma y se demostró que siguen una distribución normal. Para veríicar la estabilidad estadística se aplicó el procedimiento adaptativo del suplemento 1 de la guía GUM ISO 2008. Los cálculos se repitieron 3 veces, hasta que se obtuvo la precisión adecuada. Los valores de los parámetros finales del mensurando en $\mathrm{cm}^{2}$ fueron: $y=50.72, u_{y}=0.13, y_{\text {inf }}=50.48, y_{\text {sup }}=50.96$. También se aplicó el método clásico GUM 1995 o ley de propagación de la incertidumbre, encontrándose los siguientes valores: $y=50.72, u_{y}=0.25, y_{\text {inf }}=50.21, y_{\text {sup }}=51.22$. Al comparar los dos métodos se observó que la técnica de simulación tiene mayor precisión.

Palabras claves: incertidumbre de la medición por el método adaptativo de Monte Carlo, propagación de las distribuciones.

\section{INTRODUCCIÓN}

La evaluación de la incertidumbre en las mediciones (físicas o fisicoquímicas) aplicando la ley de propagación de la incertidumbre ya se ha discutido ampliamente en artículos anteriores $^{[1-3]}$. Pero esta ley tiene limitaciones para funciones no derivables o difícilmente derivables. La aplicación de la técnica de simulación numérica de Monte Carlo (MCM) constituye la mejor alternativa para este tipo de funciones.

Esta técnica fue aplicada en la determinación de aflatoxina B1 por HPLC con detector de fluorescencia ${ }^{[4]} y$ consiste en generar $\mathrm{N}$ variables aleatorias para obtener $\mathrm{N}$ valores de mensurando, calculando la incertidumbre como la desviación estándar. La nueva tendencia para evaluar la incertidumbre en las mediciones es aplicar el método numérico de Monte Carlo ${ }^{[5,6]}$.

Desde inicios de la presente década el Comité Adjunto para las Guías Metrológicas (JCGM) había venido trabajando en esa nueva alternativa para estimar la incertidumbre y en septiembre del año 2006 publicó un borrador del suplemento 1 a la Guía para la Expresión de la Incertidumbre GUM ISO 1995 $5^{[7]}$, titulado "Evaluation of measurement data - Suplement 1 to the Guide to the
Expression of uncertainty measurement- Propagation of distribution using a Monte Carlo Method" donde se detalla la técnica MCM con aplicaciones. En Septiembre del 2008 se publicó el documento final[8].

Los países desarrollados se ponen de acuerdo para conformar las normas que serán aplicadas en los tratados comerciales y los países pequeños deberán enmarcarse en el campo de acción de estas normas para poder exportar sus productos al país comprador. Los productos deben cumplir con las especificaciones establecidas por el cliente y para poder obtener la certificación deberán tener un informe de análisis realizado por un laboratorio acreditado, el cual deberá cumplir con los requisitos de la norma ISO/IEC $17025: 2005^{[0]}$.

Actualmente, muchos compradores o usuarios de los laboratorios de ensayos o de calibración, exigen el cumplimiento de esta norma. Los clientes exigen resultados de calidad, es decir trazables, precisos y confiables. Dentro de la precisión se exige la estimación de la incertidumbre aplicando un procedimiento documentado. 
El numeral 5.4.6 de la norma ISO/IEC 17025:2005 establece que los laboratorios de ensayo deben tener y deben aplicar procedimientos para estimar la incertidumbre de la medición ${ }^{[9]}$.

En el estudio sobre la evaluación de incertidumbre en la determinación de aflatoxina $\mathrm{B} 1^{[4]}$ se utilizó la simulación de Monte Carlo admitiendo que todas las variables de entradas eran independientes y caracterizadas por una distribución normal. El intervalo de confianza fue evaluado utilizando un factor de cobertura igual a 2 al $95.45 \%$ de nivel de confianza. Esta forma de calcular el intervalo de confianza se basó en la guía GUM ISO $1995^{[7]}$ y no se aplicó de manera estricta la técnica de simulación MCM.

En el presente trabajo se pretende ilustrar la metodología rigurosa para estimar la incertidumbre de la medición, aplicando el método adaptativo de Montecarlo del suplemento 1 de la guía GUM 2008 ${ }^{[8]}$. Esta técnica fue aplicada a la estimación de la incertidumbre de la medición del área de un triángulo, tomando como referencia los valores de los segmentos medidos en un estudio anterior ${ }^{[2]}$.

\section{TEORÍA}

Ya se ha visto que existen dos técnicas para evaluar la incertidumbre de las mediciones ${ }^{[4]}$. La primera, denominada ley de propagación de la incertidumbre, propaga las incertidumbres (las de tipo A y B) de las variables de entradas aplicando la expansión analítica de Taylor con una aproximación de primer orden (enfoque GUM) ${ }^{[6]}$ para obtener la incertidumbre en el mensurando $u_{v}$ (ecuación 1); y la segunda técnica aplica métodos numéricos de simulación de Monte Carlo $(\mathrm{MCM})^{[4,5,8]}$, en la cual se propagan las distribuciones de las variables de entradas $X=\{x i\}$ a través del modelo de medición $f\left(x_{i}\right)$ para proporcionar la distribución del resultado de la medición $y$.

La ventaja que tiene esta última técnica es que no necesita que la función sea derivable porque no es necesario calcular los coeficientes de sensibilidad, y la clasificación de las incertidumbres en tipo A y $B$ no es necesaria. En el caso de la propagación de las incertidumbres se asume que la distribución de los resultados de la medición tienen una distribución gaussiana o una $t$.

En la simulación de Monte Carlo el mensurando no está limitado a una distribución dada. Las variables de entradas pueden tener variadas distribuciones y el mensurando puede tener una distribución diferente a la normal o a la $t$. Este hecho le confiere al MCM mayor validez por obtener resultados de la medición más realistas. A continuación, se describen en forma breve los principios generales de las dos técnicas de estimación de la incertidumbre de la medición.

\section{El enfoque GUM: propagación de las incertidumbres}

Esta ley se deduce a partir de la expansión de Taylor para la función $y=f(x)$ despreciando las derivadas de segundo o más órdenes ${ }^{[4,10]}$, tomando la incertidumbre $u_{y}=\Delta y$ como la diferencia entre la función $f\left(x_{i}\right)$ y $f$ evaluada en los promedios de $x_{i}$, donde $i=1, \ldots, m$. La expresión final que representa esta ley es la siguiente:

$$
u_{(y)}^{2}=\sum\left(\frac{\partial f}{\partial x_{i}}\right)^{2} u_{x i}^{2}+2 \sum_{i=1}^{N-1} \sum_{j=i+1}^{N}\left(\frac{\partial f}{\partial x_{i}}\right)\left(\frac{\partial f}{\partial x_{j}}\right) u_{(x i, x j)}
$$

Si las variables $\left(x_{i}\right)$ son independientes, el segundo término denominado covarianza es despreciable, siendo $u\left(x_{i}, x_{j}\right)$, la incertidumbre de las variables correlacionadas. En esta ecuación se puede observar que para estimar la incertidumbre en el mesurando $y$ es necesario que la función sea derivable y además se necesita estimar los componentes de la incertidumbre las de tipo $\mathrm{A}$ y las de tipo $\mathrm{B}^{[7]}$.

Para calcular el intervalo de confianza o intervalo de cobertura, se asume una distribución normal o una distribución $t$ para un valor de grados de libertad efectivos $v_{\text {eff }}^{[2,7]}$, calculado a partir de la combinación de los grados de libertad de diferentes tipos de distribución de las variables de entradas. La incertidumbre expandida se calcula multiplicando el factor de cobertura $k$ a un nivel de probabilidad especificado por la incertidumbre del mensurando $\boldsymbol{u}_{\boldsymbol{y}}: \boldsymbol{U}=\boldsymbol{k} \times \boldsymbol{u}_{\boldsymbol{y}}$. El intervalo de confianza se expresa como: $\boldsymbol{Y}=\boldsymbol{y} \pm \boldsymbol{U}$. Los valores extremos $y_{\text {inf }}$ y $y_{\text {sup }}$ son: $\boldsymbol{y}-\boldsymbol{U}, \boldsymbol{y}+\boldsymbol{U}$.

\section{El enfoque MCM: método adaptativo de Monte Carlo}

El principio general de la técnica $\mathrm{MCM}$ consiste en generar $N(>100 /(1-p))$ valores aleatorios para cada variable de entrada $x_{j}$, generando $N$ valores del mesurando $y$, se calcula la incertidumbre estándar como desviación estándar de los $N$ valores de $y$, y por último se evalúa el intervalo de cobertura a partir de los porcentajes acumulados de la función de distribución de probabilidad (CPDF) seleccionando el valor extremo más bajo $y_{\text {inf }}$ y el más alto $y_{\text {sup }}$ con una probabilidad de $95 \%$, definido por $(1-p) / 2$ y $(1+p) / 2$ cuantiles $^{[8]}$ donde $p$ es generalmente 0.95 . La figura 1 representa el principio de la simulación numérica MCM. 


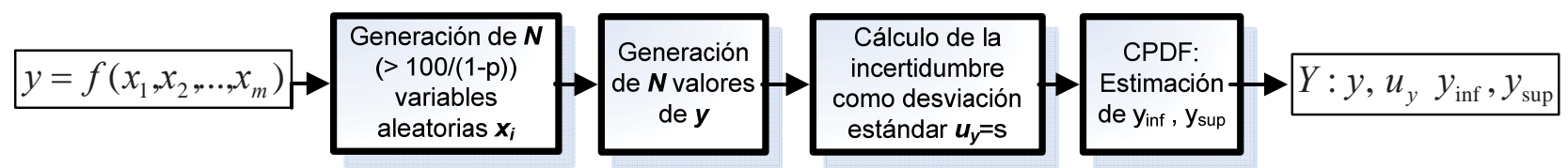

Figura 1. Principio de estimación del mensurando, su incertidumbre asociada y los valores extremos del intervalo de cobertura por la tecnica de Monte Carlo.

El método adaptativo de Monte Carlo consiste en repetir $h$ veces el algoritmo de la figura 1 hasta obtener una estabilidad estadística ${ }^{[8]}$. Este método proporciona un estimado $y$ del valor del mensurando $Y$, su incertidumbre asociada $u_{y}$, los valores extremos $y_{\text {inf }}$ y $y_{\text {sup }}$ del intervalo de cobertura para $Y$ a una probabilidad establecida (generalmente el 95\%), de tal forma que cada uno de estos cuatro valores reúnan la precisión esperada tomando como referencia la tolerancia numérica $(\delta)$ ${ }^{[8]}$ requerida. Esta tolerancia representa el número de dígitos decimales significativos de la incertidumbre en el mensurando y está dado por la siguiente expresión:

$$
\delta=\frac{1}{2} 10^{l}
$$

donde $l$ es un entero. La selección de $l$ dependerá del número de dígitos decimales significativos de la

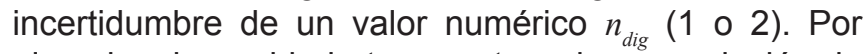
ejemplo, si se mide la temperatura de una solución de referencia de $p H$ para la calibración de un pHmetro con un termómetro caracterizado por una incertidumbre de $0.05{ }^{\circ} \mathrm{C}$, el número de dígitos significativos es $n_{d i g}=1$, y la incertidumbre se puede expresar como $5 \times 10^{l}$, donde $l=-2$, por tanto la tolerancia numérica es:

$$
\delta=\frac{1}{2} 10^{-2}=0.005
$$

\section{Etapas del método adaptativo MCM}

Las etapas para aplicar el procedimiento adaptativo de Monte Carlo ${ }^{[8]}$ son las siguientes:

a) Seleccionar el número de dígitos de decimales significativos $n_{\text {dig }}$ de la incertidumbre, generalmente 1 o 2.

b) Seleccione $N=10^{4}$.

c) Seleccione $h=1$, indicando la primer cálculo de simulación MCM.

d) Ejecute la simulación de Monte Carlo según el algoritmo de la figura 4 para $N$ seleccionado.

e) A partir de los $N$ valores de $y\left(y_{1}, \ldots, y_{N}\right)$ calcular el promedio de los $N$ valores de $y$, designándolo $y^{(l)}$. Calcule la incertidumbre $u y^{(l)}$, y los valores límites del intervalo de cobertura $y^{(l)}{ }_{i n f}, y^{(l)}{ }_{\text {sup }}$ para $2.5 \%$ y $97.5 \%$ respectivamente.

f) Si $h=1$, incrementar $h$ en una unidad y regresar a la etapa $d$ ).

g) Calcular el promedio $y$ de los valores $y^{(l)}, \ldots, y^{(h)}$ y la desviación estándar asociada.

24 | Universitas 3 [2] 2009, ISSN 2071-2575 h) De igual manera calcular los promedios de $u_{(y)}, y_{i n f}$ y $y_{\text {sup }}$ desde $l$ hasta $h$.

i) Calcular la tolerancia numérica $\delta$ asociada con $u_{(v)}$.

j) Si cualquiera de las siguientes condiciones $2 s_{y}$, $2 s_{u(v)}, 2 s_{\text {yinf }} 2 s_{\text {ysup }}$ es mayor que $\delta$ incremente $h$ en una unidad y continúe con la etapa $d$ ).

k) Una vez obtenida la estabilidad estadística, considere todos los valores del modelo $h \times N$ para estimar $y, u_{(y)}, y_{\text {inf }} y_{\text {sup }}$, para $95 \%$ de probabilidad.

\section{Generación de valores aleatorios según el tipo de distribución y su propagación}

Cuando una variable de entrada $x_{j}$ es afectada por diferentes tipos de distribuciones (uniforme, triangular, normal, etc.) se deben generar los valores aleatorios $\left(\varepsilon_{j}\right)$ correspondiente a cada distribución; el promedio de los valores $\varepsilon_{j}$ generados es el valor aleatorio de la variable $x_{i}$. Existen diferentes distribuciones para las variables aleatorias $^{[7,8]}$ : rectangular, triangular, trapezoidal, normal, Arco seno o $U$, $t$, gamma.

Entre las distribuciones más comunes en las mediciones químicas y físicas están la normal, $t$, rectangular y triangular. Como ejemplo, se tiene la medición de un segmento de recta $\left(x_{i}\right)$ con una regla graduada. La medición está influenciada por la distribución $t$ en $n$ réplicas de lecturas y por una distribución uniforme o rectangular debido a la resolución de la regla ${ }^{[2]}$. El esquema de la figura 2 representa la propagación de los dos tipos de incertidumbre en la variable $x_{j}$.

El valor aleatorio generado para $x j$ con una distribución $t$, se obtiene según la ecuación (2) ${ }^{[8]}$. El valor de $t_{v}$ se obtiene aleatoriamente entre $-t_{v} \mathrm{y}+t_{v}$, utilizando la función RandStudentst en el programa de cálculo simbólico Maple 11, para $v=n-1$ grados de libertad. Donde $s$ es la desviación estándar para $n$ réplicas de lecturas.

$$
\varepsilon_{1}=x_{j}+t_{v} \sqrt{\frac{v}{v-2}} \frac{s}{\sqrt{n}}
$$




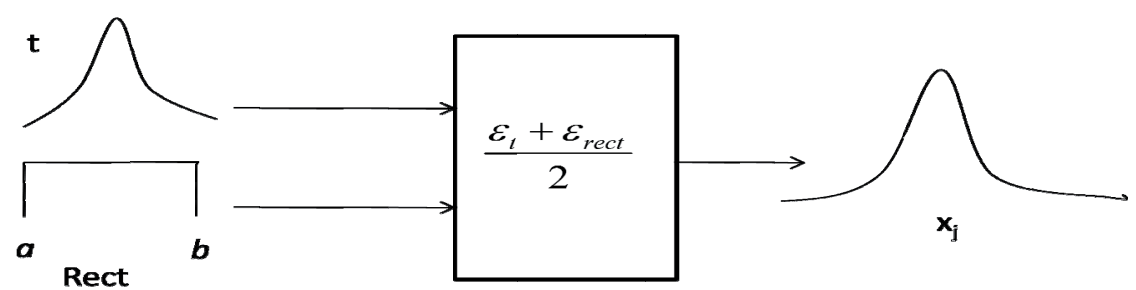

Figura 2. Propagación de las distribuciones $t$ y rectangular en la variable $x_{j}$.

Para una distribución rectangular el valor aleatorio generado para $x_{j}$ se obtiene con la ecuación (3).

$$
\varepsilon_{2}=a+(b-a) r_{i}
$$

Donde $a$ y $b$ son los valores extremos de la resolución $\mathrm{R}$ de la regla, calculados según las ecuaciones (4) y (5). El valor aleatorio $r_{i}$ entre 0 y 1 se obtiene con la función RandUniform en el programa Maple.

$$
\begin{aligned}
& a=x_{j}-\frac{R}{2} \\
& b=x_{j}+\frac{R}{2}
\end{aligned}
$$

La propagación de las dos distribuciones sobre la variable $x_{j}$ se obtiene el valor aleatorio que se expresa como (6).

$$
v_{j}=\frac{\varepsilon_{1}+\varepsilon_{2}}{2}
$$

Si se tiene el modelo $y=f\left(x_{0}, x_{1}, x_{2}, x_{3}\right)$, se pueden generar valores aleatorios de cada variable de entrada $v_{j}: v_{0^{\prime}}, v_{l}$, $v_{2}, v_{3}$. Se calcula el mesurando $y$ y se relacionan las variables con la función $f(v)$. Si se repite el proceso $N$ veces se obtiene la función $y_{i}=f\left(v_{i i}\right)$ y se generan $N$ valores del mesurando desde $y_{1}$, hasta $y_{N}$.

\section{PARTE EXPERIMENTAL}

\section{Materiales}

-Computadora equipada con el programa de cálculo simbólico MAPLE 12.

- Instrumento de medición: regla calibrada de madera impermeabilizada, graduada en centímetro, con resolución de $0.05 \mathrm{~cm}$ y $0.30 \mathrm{~m}$ de longitud.

- Objetos de medición: un triángulo con la base representada por los segmentos $x_{1}$ y $x_{3}$ y la altura por $x_{2}$. Ver anexo.

\section{Procedimiento}

El procedimiento para las mediciones de los segmentos del triángulo se describe en un estudio anterior ${ }^{[2]}$. Las mediciones fueron realizadas por 10 estudiantes de la Maestría en Química y Gestión de la Calidad. Cada estudiante midió con la misma regla los segmentos de la figura geométrica, ubicando con precisión el cero con el extremo izquierdo de la regla y el valor del segmento a medir con el extremo derecho. Las mediciones fueron realizadas tratando de minimizar el error de paralaje y los resultados se registraron en una matriz de datos. La variación de temperatura durante el proceso de medición fue de $2^{\circ} \mathrm{C}$.

\section{RESULTADOS Y DISCUSIÓN}

Los resultados de las mediciones de los segmentos de la figura geométrica se presentan en la tabla 1.

TABLA 1. Resultados de la medición de los segmentos de las figuras geométricas en $\mathrm{cm}$. Los segmentos se simbolizan por $x_{i}$. En todas las mediciones se toma en cuenta la lectura del punto 0.00 de la regla, simbolizado como $x_{0}$.

\begin{tabular}{|c|c|c|c|}
\hline Estudiante & \multicolumn{3}{|c|}{ Segmentos del triángulo en cm } \\
\hline$N^{\circ}$ & $x_{1}$ & $x_{2}$ & $x_{3}$ \\
\hline 1 & 8.30 & 7.85 & 4.55 \\
\hline 2 & 8.30 & 7.90 & 4.60 \\
\hline 3 & 8.30 & 7.90 & 4.60 \\
\hline 4 & 8.30 & 7.90 & 4.60 \\
\hline 5 & 8.25 & 7.90 & 4.60 \\
\hline 6 & 8.25 & 7.85 & 4.60 \\
\hline 7 & 8.30 & 7.85 & 4.60 \\
\hline 8 & 8.30 & 7.90 & 4.55 \\
\hline 9 & 8.30 & 7.90 & 4.55 \\
\hline 10 & 8.25 & 7.90 & 4.60 \\
\hline $\bar{x}_{\mathrm{j}}$ & 8.285 & 7.885 & 4.585 \\
\hline$s_{x j}$ & 0.02415229 & 0.02415229 & 0.02108185 \\
\hline
\end{tabular}


Para calcular la incertidumbre en la medición del área del triángulo se aplicó el procedimiento basado en las etapas de la simulación del método adaptativo de Montecarlo MCM descrito anteriormente.

El procedimiento se inicia con la definición del mesurando, se identifican las variables que influyen, se deduce el modelo matemático, se identifican las distribuciones de cada una de las variables de entradas (normal, t, rectangular, triangular, etc.), se selecciona el número de valores generados aleatoriamente $(N=10000)$ y se generan $N$ variables aleatorias $x_{j}$, se aplica el modelo para relacionar las variables $y=f\left(x_{j}\right)$, se generan $N$ valores del mesurando $y_{1} \ldots y_{10000}$, se calcula el promedio de $N$ valores de $y$, se calcula su incertidumbre $u_{y}$ como desviación estándar:

$$
u_{y}=\sqrt{\frac{\sum_{1}^{N}\left(y_{i}-\bar{y}\right)^{2}}{N-1}}
$$

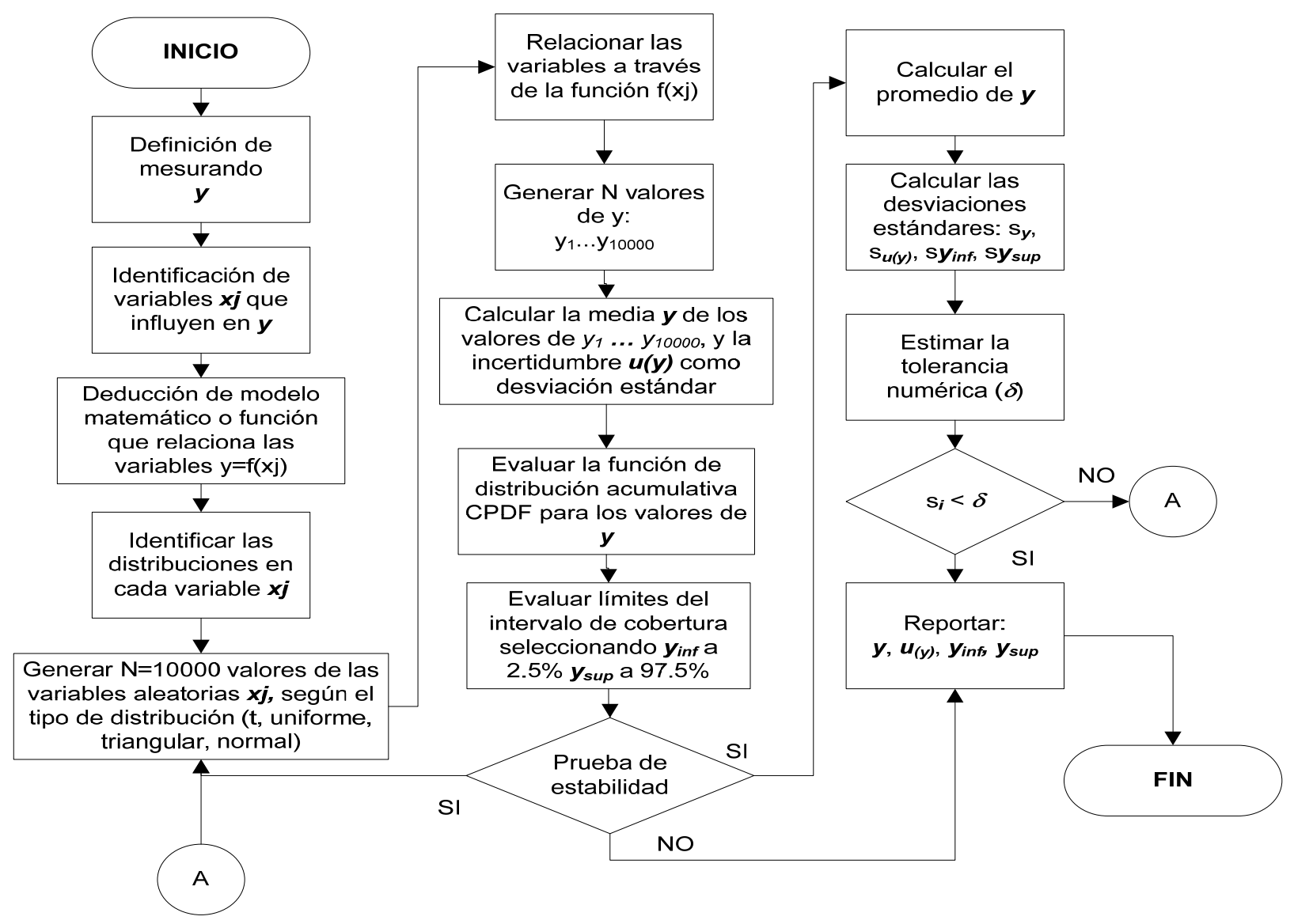

Figura 2. Diagrama de flujo que describe el método adaptativo de Montecarlo para estimar el valor más probable del mensurando $y$, su incertidumbre $u_{y}$, y los valores extremos inferior $\left(y_{\text {inf }}\right)$ y superior $\left(y_{\text {sup }}\right)$ del intervalo de confianza o de cobertura para un $95 \%$. 


\section{APLICACIÓN DEL MÉTODO ADAPTATIVO MCM}

\section{Definición del mesurando y de las variables que influyen}

El área del triángulo $A B C$ (Anexo) es el mensurando, designándolo con la letra $y$, y las variables que influyen son la base, formada por los segmentos $x_{1}$ y $x_{3}$, y la altura representada por el segmento $x_{2}$. En cada medición se toma cuenta la lectura del punto $x_{0}$ del extremo inferior del segmento.

\section{Modelo matemático}

El área del triángulo es calculada multiplicando la base por la altura dividido entre 2. La expresión matemática está representada en la ecuación (7).

$$
y=f\left(x_{0}, x_{1}, x_{2}, x_{3}\right)=\frac{\left(x_{1}+x_{3}-2 x_{0}\right)\left(x_{2}-x_{0}\right)}{2}
$$

\section{Identificación de los tipos de distribuciones de las variables de entradas $x_{0}, x_{1}, x_{2}, x_{3}$}

Cada medición del segmento $x_{i}$, tiene dos tipos de distribuciones, distribución $t$ debido a las mediciones de $n$ lecturas y una distribución rectangular debido a la resolución de la regla graduada en $\mathrm{cm}$. En la figura 3 se presentan las diferentes distribuciones de las variables $x_{j}$ que influyen en la función de distribución de probabilidad (PDF) del mesurando $y$. Para generar los valores aleatorios de las variables se toman en cuenta estas dos clases de distribuciones, utilizando las ecuaciones (2) y (3).

\section{Estimación del área del triángulo y su incertidumbre usando Maple}

Anteriormente ${ }^{[4]}$ se elaboró el programa de simulación numérica de Montecarlo en lenguaje Maple, tomando en cuenta que la distribución de las variables de entradas y la del mesurando obedecen a la normal. En el presente estudio, se elaboró el programa para calcular el área del triángulo (o mensurando $y$ ) y la incertidumbre $\left(u_{y}\right)$, tomando en cuenta que cada variable $x_{j}$ obedece a dos distribuciones: $t$ y rectangular.

El algoritmo está basado en el diagrama de flujo de la figura 2 y en las etapas del método adaptativo de Montecarlo. Se generan $N=10000$ valores aleatorios de cada una de las variables de entradas $x j$ aplicando las ecuaciones (2) a (6), se relacionan las variables a través de la función del modelo de la ecuación (7) y se obtienen los 10000 valores de $y_{i}$.

Se calculan el promedio de $y_{1} \ldots y_{10000}$ para estimar el área del triángulo (y) y la desviación estándar para evaluar la incertidumbre $u_{y}$. El programa base se muestra en la figura 4 .

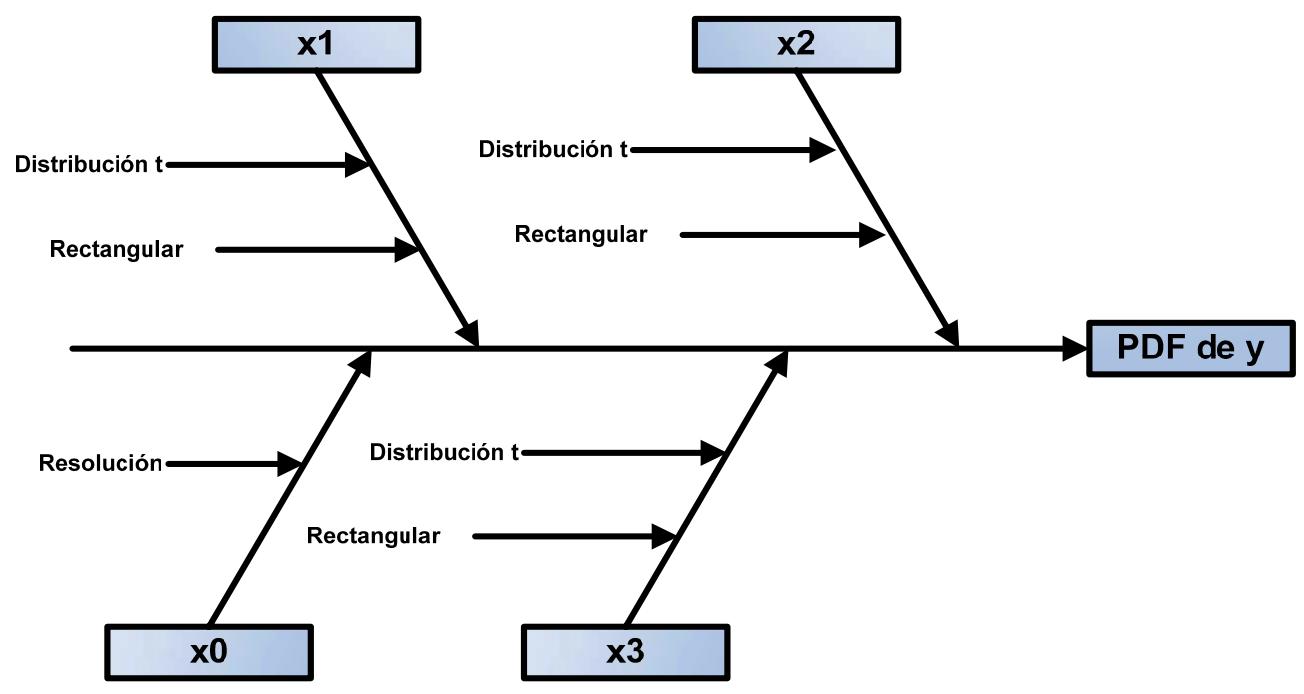

Figura 3. Tipos de distribución de en cada uno de las variables de entradas $x_{0}, x_{1}, x_{2} y x_{3}$, que influyen en la función de distribución de probabilidad (PDF) del mensurando $y$. 

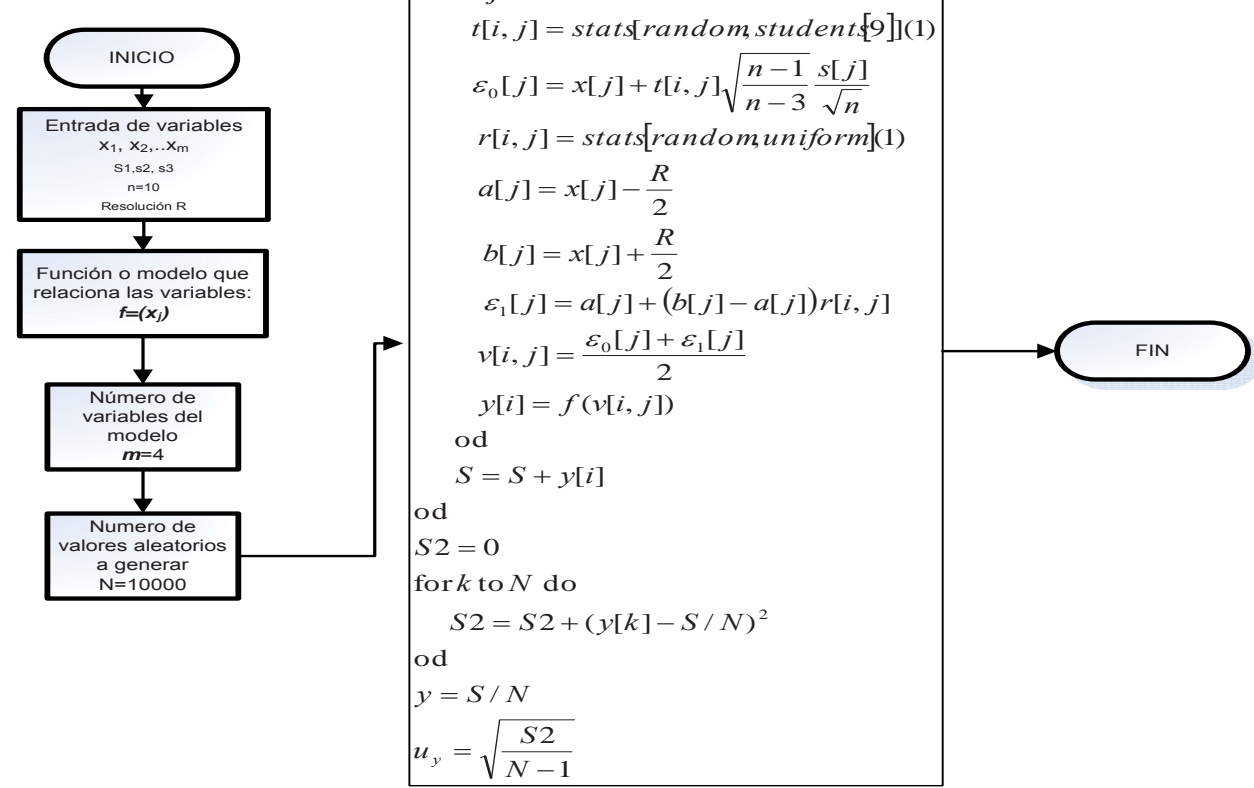

Figura 4. Programa en Maple para estimar el valor más probable del área del triángulo (y) y de la incertidumbre estándar $u_{y}$.

Los datos introducidos en el programa anterior se resumen en la tabla 2.

\begin{tabular}{cccc}
\hline Variable $x j$ & Valor & $s_{j}$ & Resolución $R^{*}$ \\
\hline$x_{0}$ & 0.000 & 0.000 & 0.05 \\
$x_{1}$ & 8.285 & 0.02415229 & 0.05 \\
$x_{2}$ & 7.885 & 0.02415229 & 0.05 \\
$x_{3}$ & 4.585 & 0.02108185 & 0.05 \\
\hline
\end{tabular}

(*) Resolución se define como el mínimo valor leído[10].

Los valores estimados del área $(y)$ del triángulo y de su incertidumbre estándar $u_{v}$ en $\mathrm{cm}^{2}$ son:

\begin{tabular}{|c|c|}
\hline$y$ & $u_{y}$ \\
\hline 50.7172271 & 0.12699204 \\
\hline
\end{tabular}

Estimación de los valores límites del intervalo de cobertura usando Excel: $y_{\text {inp }} y_{\text {sup }}$

Los 10000 valores de $y_{i}$ generados en Maple fueron exportados a Excel para obtener la función de distribución de probabilidad acumulativa (CPDF), seleccionando el valor extremo más bajo $y_{\text {inf }}$ y el más alto $y_{\text {sup }}$ con una probabilidad de 95\%,. La figura 5 muestra la curva CPDF, indicando el intervalo de cobertura definido por $2.5 \%$ y $97.5 \%$ cuantiles. Los valores en $\mathrm{cm}^{2}$ son 50.4778026 para $y_{\text {inf }}$ y 50.9577188 para $y_{\text {sup }}$. El valor de $y_{\text {sup }}$ fue calculado por interpolación lineal inversa ${ }^{[8]}$, utilizando la ecuación (8).

$$
y_{\text {sup }}=y_{s}+\left(y_{s+1}-y_{s}\right) \frac{p-p_{s}}{p_{s+1}-p_{s}}
$$

Los resultados obtenidos en Excel de los porcentajes acumulados de la función de distribución de probabilidad (CPDF) para calcular el $y_{\text {sup }}$ son los siguientes: $y_{s}=50.9567422$ es el valor de $y$ para el porcentaje acumulado $p_{s}$ (0.974) menor que $p=0.975$ (97.5\%); $y_{s+1}$ (50.9586955) es el valor de $y$ para $p_{s+1}(0.976)$ mayor que p.

\section{CPDF para Area de Triángulo}

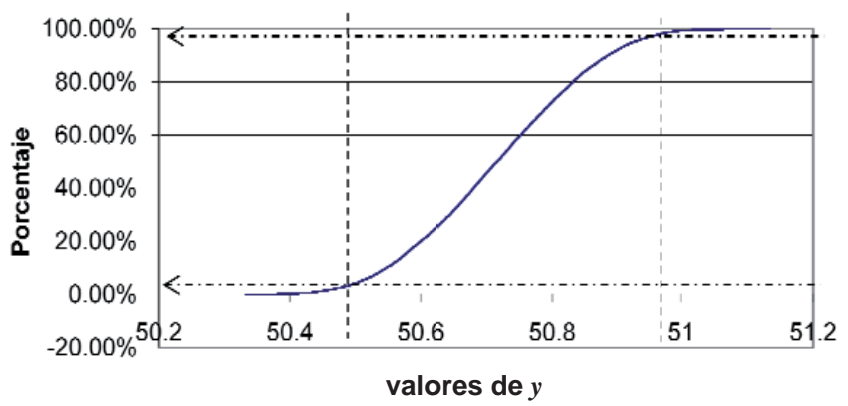

Figura 5. Porcentajes acumulados de la función de distribución de probabilidad en función de los valores de $y$ en $\mathrm{cm}^{2}$. Las líneas punteadas representan el intervalo de cobertura limitada por los extremos inferior y superior: $y_{\text {inf }}=50.48, y_{\text {sup }}=50.96$. 


\section{Curva de distribución del mensurando y}

El histograma, obtenido con Excel, de los resultados de los 10000 valores de $y$ se muestran en la figura 6 . De acuerdo a la gráfica, se observa la simetría de la distribución normal. Para verificar si los resultados de la medición del área del triángulo sigue una distribución normal, se efectuó el ajuste de Kolmogorov-Smirnov utilizando el paquete estadístico STATGRAPHICS, encontrándose que la distribución es normal con un nivel de confianza mayor o igual al $90 \%$.

Este resultado es congruente con la forma de la gráfica CPDF de la figura 5 . Se observa un sigmoide muy simétrico. Es necesario remarcar que la distribución del mesurando no necesariamente tiene que ser una normal, puede tener cualquier otra distribución. Lo único que interesa es el intervalo de cobertura $\left(y_{\text {inp }}, y_{\text {sup }}\right)$ con $(1-p) / 2$ y $(1+p) / 2$ cuantiles.

\section{Histograma}

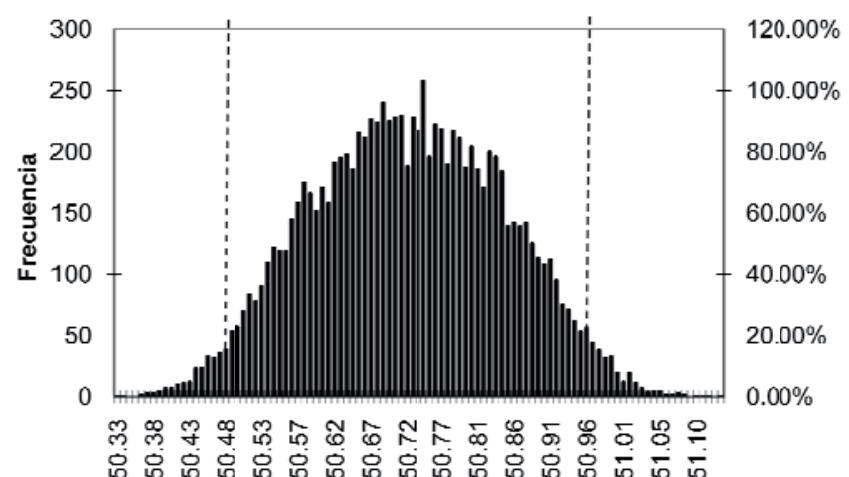

Clase

Figura 6. Histograma de los resultados de los 10000 valores del mesurando en $\mathrm{cm}^{2}$ obtenidos por la simulación numérica de Monte Carlo. Las líneas punteadas es el intervalo de cobertura para $2.5 \%$ y $97.5 \%$ quantiles.

\section{Cálculo de la tolerancia numérica $(\delta)$}

La incertidumbre $u_{v}$ obtenida por la simulación se puede expresar como $z=13 \times 10^{-2}$, tomando $n_{\text {dig }}=2$ como el número de dígitos significativos. La tolerancia numérica asociada a $z$ es:

$$
\delta=\frac{1}{2} 10^{-2}=0.005
$$

\section{Prueba de la estabilidad estadística}

Para demostrar la precisión en los cálculos es necesario comparar las desviaciones estándares de $h$ veces el cálculo de $y, u_{v}, y_{i n \rho} y_{\text {sup }}$. Si el doble de cualquiera de estas desviaciones $\left(2 s_{h}\right)$ es mayor que la tolerancia numérica $(\delta)$ repetir los cálculos hasta obtener un valor menor que 0.005 . Para el presenta caso, bastaron solamente 3 ciclos $(h=3)$ de cálculos para obtener la estabilidad. La tabla 3 muestra el resumen de los resultados. Se observa que 2 veces las desviaciones estándares de todos los parámetros característicos del mesurando son menores que la tolerancia numérica.

Tabla 3. Parámetros característicos del mesurando para 3 ciclos de 10000 valores del área del triángulo en $\mathrm{cm}^{2}$ por simulación MCM.

\begin{tabular}{|c|c|c|c|c|}
\hline$h$ & $y$ & $u_{y}$ & $y_{\text {inf }}$ & $y_{\text {sup }}$ \\
\hline 1 & 50.7172271 & 0.12699204 & 50.4778026 & 50.9577188 \\
\hline 2 & 50.71748 & 0.12699179 & 50.482413 & 50.9557292 \\
\hline 3 & 50.7162868 & 0.12859557 & 50.478711 & 50.9568996 \\
\hline promedio & 50.716998 & 0.12699192 & 50.4801078 & 50.956724 \\
\hline $\begin{array}{c}\text { desv est. } \\
s(h)\end{array}$ & 0.00062876 & 0.00092587 & 0.0024422 & 0.00099997 \\
\hline $2 s(h)$ & 0.00125752 & 0.00185174 & 0.0048844 & 0.00199993 \\
\hline
\end{tabular}

Los parámetros finales con los dígitos significativos son los promedios de la tabla 4 y se resumen a continuación:

- Área del triángulo (mesurando):

$y=50.72 \mathrm{~cm}^{2}$

- Incertidumbre estándar:

$$
u_{y}=0.13 \mathrm{~cm}^{2}
$$

- Límite inferior del intervalo de cobertura:

$$
y_{\text {inf }}=50.48 \mathrm{~cm}^{2}
$$

- Límite superior del intervalo de cobertura:

$$
y_{\text {sup }}=50.96 \mathrm{~cm}^{2}
$$

- Rango del intervalo cobertura:

$$
R I C=y_{\text {sup }}-y_{\text {inf }}=0.48 \mathrm{~cm}^{2}
$$

\section{Comparación de los resultados con el método clásico GUM 1995}

Los datos necesarios para el cálculo de la incertidumbre $u$ por el método GUM aplicando la ecuación (1) se encuentran en la tabla 4. Se utilizaron las ecuaciones (9) y (10) para el cálculo de las incertidumbres $u_{x j}$ de las variables $x_{j}$ y de los coeficientes de sensibilidad $c_{x j}$ considerando $n=10$ lecturas repetidas.

$$
\begin{array}{r}
u_{x j}=\sqrt{\frac{R^{2}}{12}+\frac{n-1}{n-3} \frac{s_{x j}^{2}}{n}} \\
c_{x j}=\frac{\partial y}{\partial x_{j}}
\end{array}
$$


Tabla 4. Valores de incertidumbres de xj y de los coeficientes de sensibilidad.

\begin{tabular}{ccc}
\hline$x_{j}$ & $u_{x j}$ & $c_{x j}$ \\
\hline$x_{0}$ & 0.01443375672 & -14.3170 \\
$x_{1}$ & 0.01674358014 & 3.9425 \\
$x_{2}$ & 0.01683250738 & 6.4320 \\
$x_{3}$ & 0.01714781693 & 3.9425 \\
\hline
\end{tabular}

El cálculo de $u_{y}$ aplicando la ecuación (1) y considerando las variables $x_{j}$ independientes, se detalla en la ecuación (11).

$$
u_{y}=\sqrt{\left(c_{x 0} u_{x 0}\right)^{2}+\left(c_{x 1} u_{x 1}\right)^{2}+\left(c_{x 2} u_{x 2}\right)^{2}+\left(c_{x 3} u_{x 3}\right)^{2}}
$$

Los valores característicos del mesurando $\left(y, u, y_{\text {inf }} y_{\text {sup }}\right)$ obtenidos por la propagación de las incertidumbres y los obtenidos por el método MCM se presentan en la tabla 5. Se observa que con la simulación numérica de Montecarlo se obtienen incertidumbres menores, tal a como se comprueba en el rango del intervalo de cobertura (RIC) al 95\% de nivel de confianza, 1.00 para el método GUM 1995 y 0.48 para el método de Montecarlo.

Tabla 5. Resultados de los valores característicos del mesurando en $\mathrm{cm}^{2}$ por el método GUM 1995 y el método MCM.

\begin{tabular}{cccccc}
\hline Método & $y$ & $u_{y}$ & $y_{\text {inf }}$ & $y_{\text {sup }}$ & RIC \\
\hline $\begin{array}{c}\text { GUM } \\
1995^{[2,7]}\end{array}$ & 50.72 & 0.25 & 50.21 & 51.22 & 1.00 \\
$M C M$ & 50.72 & 0.13 & 50.48 & 50.96 & 0.48 \\
\hline
\end{tabular}

Una de las ventajas que tiene el método MCM es que sirve para validar cualquier otro proceso de evaluación de la incertidumbre de la medición. Si se quiere validar el método clásico GUM 1995 se debe cumplir las siguientes relaciones

$$
\begin{aligned}
& d_{\text {inf }}=\left|y-U-y_{\text {inf }}\right| \leq \delta \\
& d_{\text {sup }}=\left|y+U-y_{\text {sup }}\right| \leq \delta
\end{aligned}
$$

Donde $y(=50.72)$ y $U(=2 \times 0.25)$ son el área del triángulo y su incertidumbre expandida obtenido por el método GUM 1995. Los resultados son $d_{\text {inf }}=0.27 \mathrm{y} d_{\text {sup }}=0.26 \mathrm{y}$ son mayores que $\delta=0.005$.

En los dos casos no se cumplen las relaciones y se concluye que para el presente estudio el método MCM proporciona resultados más precisos que el enfoque GUM para un nivel de confianza del 95\%.

\section{Contribución de las variables a la incertidumbre de la medición del área $u_{y}$}

Para evaluar las variables que más aportan a la incertidumbre del mesurando $u_{y}$ se utiliza el enfoque GUM a través de los coeficientes de sensibilidad y de las incertidumbres de las variables, usualmente presentados en términos de "presupuesto de las incertidumbres"[2,7].

No obstante, ni la propagación de las distribuciones ni su implementación utilizando el método MCM no proporcionan los coeficientes de sensibilidad. El nuevo suplemento de la guía GUM 2008, no explica claramente la forma de identificar la variable predominante que contribuye a $u_{v}$ asociada con el estimado del mesurando $y$.

En el apartado $B$ de la guía ${ }^{[8]}$ se comenta que es posible evaluar los coeficientes de sensibilidad sin aproximaciones, calculando la razón entre la desviación estándar de los valores del modelo y la incertidumbre $u_{v}$. Por tanto, para identificar la variable que más contribuye a $u_{y}$, el enfoque GUM es de gran utilidad. Aplicando la ecuación (12 $)^{[2]}$ se puede obtener el índice de contribución como porcentaje.

$$
\operatorname{Ind} \%=\frac{\left|c_{x j} u_{x j}\right|}{\sum\left|c_{x j} u_{x j}\right|} \times 100
$$

Sustituyendo los valores de la tabla $\mathrm{N}^{\circ} 4$ en la ecuación anterior y graficando Ind\% para cada variable $x_{j}\left(x_{0}, x_{1}\right.$, $x_{2}, x_{3}$ ) se tiene el diagrama de Pareto de la figura 7.

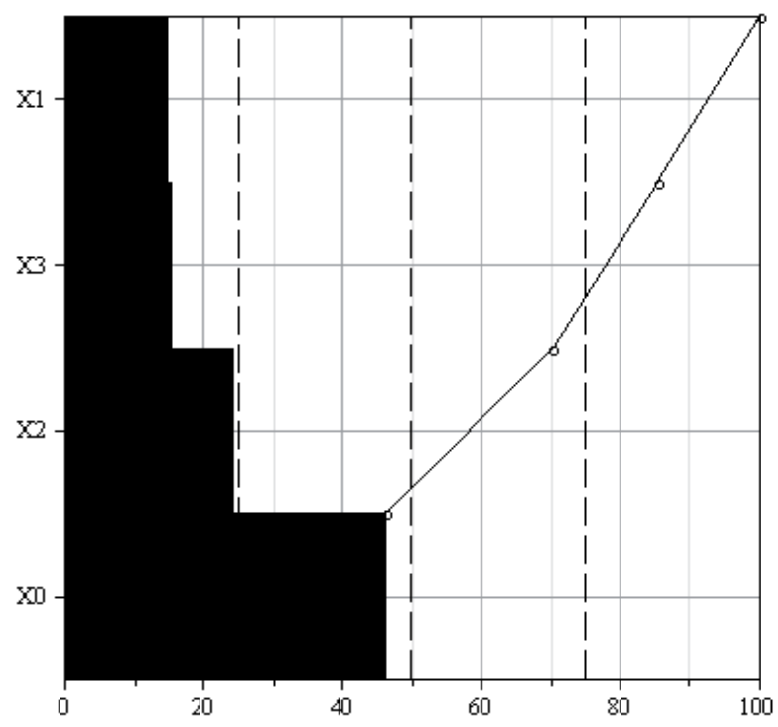

Figura 7. Diagrama de Pareto, obtenido en Maple 12, para la contribución de las variables de entrada $\left(x_{0}, x_{2}, x_{3}, x_{1}\right)$ a la incertidumbre en la medición del área del triángulo $u_{y}$. 
De acuerdo al diagrama, se observa que la precisión en la medición del 0.00 de la regla graduada es la que contribuye más a la incertidumbre en el mesurando. Esto significa que para poder disminuir la incertidumbre es necesario utilizar un instrumento de medición con menor valor de resolución en la escala, es decir mayor precisión de lectura.

\section{CONCLUSIONES}

Se demostró que la propagación de las distribuciones, utilizando el método adaptativo de Monte Carlo, tiene mayor precisión en el cálculo de la incertidumbre de las mediciones que el método clásico o enfoque GUM de la propagación de las incertidumbres. Esto se confirmó al comparar el intervalo de cobertura calculado en la medición del área de un triángulo aplicando el método MCM y el enfoque GUM. La longitud del intervalo o el rango fue de 0.48 para $\mathrm{MCM}$ y 1.00 para el enfoque GUM. Estas diferencias fueron significativas al comparar la diferencia de los valores extremos del intervalo de los dos métodos con la tolerancia numérica.

Dado que el método MCM no proporciona los coeficientes de sensibilidad, se aplicó el enfoque GUM para identificar y cuantificar la contribución de la variable que más influye en la medición del área del triángulo. A través del diagrama de Pareto, se demostró que la resolución de la graduación de la regla en la medición del $0.00 \mathrm{~cm}$ es el que más contribuye a la incertidumbre del mesurando. Para mejorar la precisión en el cálculo del área es necesario utilizar una regla con un valor de resolución menor a $0.05 \mathrm{~cm}$.

Por ser un método probabilístico, la técnica Monte Carlo aplicada al cálculo de la incertidumbre y del intervalo de confianza del mesurando, puede ser utilizada como un método de referencia en la validación de otras formas de evaluar la incertidumbre. El ejemplo del cálculo del área de un triángulo, sirvió para ilustrar la metodología rigurosa que puede seguirse en la estimación de la incertidumbre de las mediciones físicas o químicas en los laboratorios de ensayos y de calibración.

\section{AGRADECIMIENTO}

El autor agradece al Dr. Bertram Nagel, Consultor PTB, Leipzig, Alemania, por sus aportes en la elección del ejemplo, lo que sirvió para ilustrar la aplicación de la metodología del suplemento 1 de la GUM 2008, como una nueva alternativa para evaluar la incertidumbre.

\section{REFERENCIAS BIBLIOGRÁFICAS}

1. DELGADO M., VANEGAS M. Y DELGADO G., (2008) "Metrología Química II: Estimación de la incertidumbre en la medición de $\mathrm{pH}$ en aguas", Universitas UNAN-León, Vol 1, 2, 7-17

2. DELGADO G y NAGEL B. (2008) "Un experimento sencillo para evaluar la incertidumbre siguiendo la guía GUM ISO 1995 y utilizando el cálculo simbólico MAPLE 11.0", Universitas UNAN-León, Vol 1, 2, 1926.

3. DELGADO G. y HERNÁNDEZ N., (2009), "Estimación de la incertidumbre en la determinación de aflatoxina B1 en maní de exportación por HPLCFD”, Universitas UNAN-León, Vol. 3, 1, 5-15.

4. DELGADO G. y HERNÁNDEZ N., (2009), "Cálculo de la incertidumbre por simulación de Montecarlo en la determinación de aflatoxina B1 en maní de exportación por HPLC-FD. Aplicación a la evaluación de la conformidad", Universitas UNANLeón, Vol. 3, 1, 16-26.

5. HERRADOR, M.A., GONZÁLEZ G.A., (2004), "Evaluation of Measurement uncertainty in Analytical Assayes by means of Monte Carlo Simulation", Talanta, Vol. 64, 6, 415-422.

6. COWELLD.A., HOLKOMKE J.A., (1990), "Analytical applications of Monte Carlo”, Anal. Chem., 62, 529A-542A.

7. BIPM, IEC, IFCC, IUPAC, OIML (1995:2008). Guide for to the Expression of Uncertainty in Measurement (GUM), ISO, Ginebra.

8. a) BIPM, IEC, IFCC, IUPAC, ILAC, ISO, IUPAP y OIML, (2006). "Suplement 1 to the Guide for to the Expression of Uncertainty in Measurement (GUM). Propagation of the distributions using a Monte Carlo Method" (Draft), Paris. b) BIPM, IEC, IFCC, IUPAC, ILAC, ISO, IUPAP y OIML, (2008). "Evaluation of measurements data. Supplement 1 to the Guide for to the Expression of Uncertainty in Measurement (GUM). Propagation of the distributions using a Monte Carlo Method", Paris.

9. ISO/IEC 17025(ES) (2005). "Requisitos Generales para la Competencia de los Laboratorios de Ensayo y de Calibración", Norma Internacional, Ginebra, Segunda Edición. 
10. KIMOTHI, S.K., (2002), The Uncertainty of Measurements, ASQ Quality Press, Wisconsin , pag. 205.

\section{ANEXO}

La escala de figura original utilizada durante el experimento variar de MS Word a la configuración del editor de texto utilizado en la revista científica.

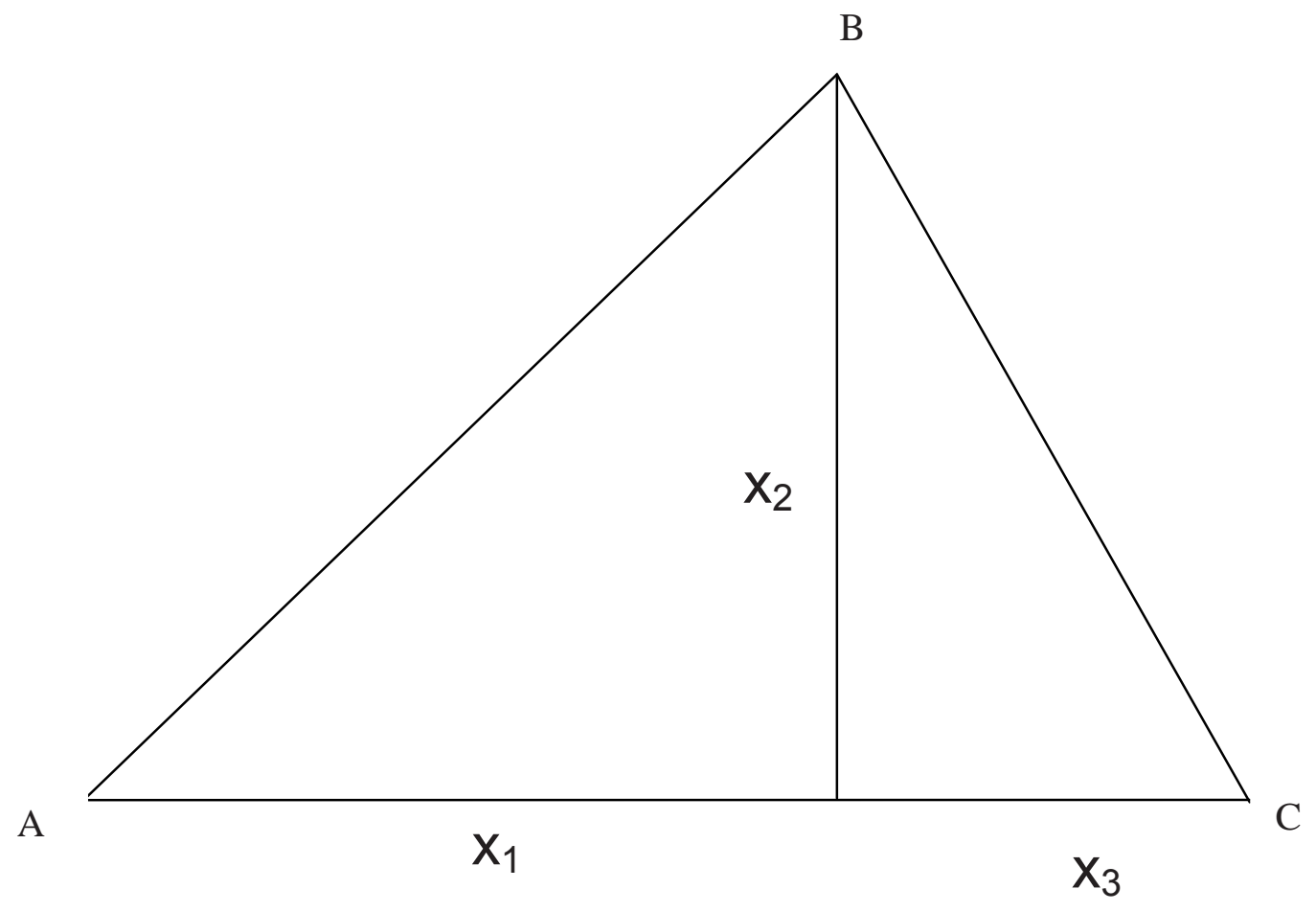

